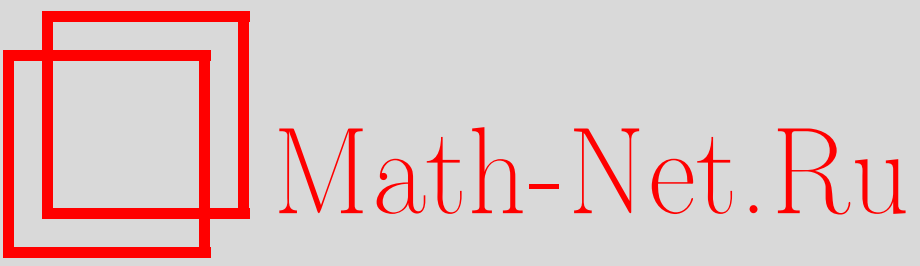

Е. И. Михайловский, Е. В. Тулубенская, Алгоритм движения по параметру жёсткости в проблеме устойчивости на границе винклеровых сред, Вестн. Сам. гос. техн. ун-та. Сер. Физ.-мат. науки, 2011, выпуск 3(), 62-71

DOI: https://doi.org/10.14498/vsgtu843

Использование Общероссийского математического портала Math-Net.Ru подразумевает, что вы прочитали и согласны с пользовательским соглашением http: //www. mathnet.ru/rus/agreement

Параметры загрузки:

IP : 3.85 .5 .30

26 апреля 2023 г., $10: 29: 36$ 
УДК 539.3

\section{АЛГОРИТМ ДВИЖЕНИЯ ПО ПАРАМЕТРУ ЖЁСТКОСТИ В ПРОБЛЕМЕ УСТОЙЧИВОСТИ НА ГРАНИЦЕ ВИНКЛЕРОВЫХ СРЕД}

\section{Е. И. Михайловский, Е.В. Тулубенская}

Сыктывкарский государственный университет, математический факультет, 167001, Сыктывкар, Октябрьский пр., 55.

E-mails: emich@inbox.ru, tulubenskaya@bk.ru

Для решения проблемы устойчивости одномерных элементов конструкиий на граниче разномодульных упругих сред предложен алгоритм движения по параметру жёсткости одной из сред. Исследована эволюиия трёхполуволновой собственной формы продольно сжимаемого стержня при граничных условиях шарнирного опирания и жёсткой заделки с учётом поперечных сдвигов по модели С.П. Тимошенко.

Ключевые слова: устойчивость, спектралъная задача, перебор вариантов.

Введение. Задачи на устойчивость одномерных элементов конструкций на границе разномодульных упругих сред винклерова типа при использовании конечно-разностной аппроксимации (на сетке размерностью $m$ ) приводятся к решению спектрального уравнения

$$
A \tilde{w}+C \tilde{w}=\lambda Q \tilde{w}
$$

где $A, Q$ - ленточные положительно определённые матрицы $(m-1) \times(m-1)$; $\tilde{w}=\left[w_{1}, w_{2}, \ldots, w_{m-1}\right]^{\top}, w_{i}=w\left(\xi_{i}\right), \xi_{i}=h i, h=\pi / m ; b=\left[b_{1}, b_{2}, \ldots, b_{m-1}\right]^{\top}$, $b$ - вектор формы такой, что

$$
b_{i}= \begin{cases}1, & w>0 \\ 0, & w \leqslant 0\end{cases}
$$

$C=h \operatorname{diag}\left[c_{1}\left(k_{1} b_{1}+k_{2}\left(1-b_{1}\right)\right), \ldots, c_{m-1}\left(k_{1} b_{m-1}+k_{2}\left(1-b_{m-1}\right)\right)\right]$ - матрица жёсткостей, $k_{1}, k_{2}$-безразмерные параметры жёсткости; $\lambda$ - собственное число.

Граничные условия задачи отражены в структурах матриц $A$ и $Q$.

Спектральная задача (1) является неопределённой из-за того, что компоненты $b_{i}, i \in\{1,2, \ldots, m-1\}$, вектора формы изначально неизвестны. Можно использовать следующий алгоритм нахождения первой собственной пары (числа и формы) уравнения (1). Задаётся некоторый вектор формы и решается соответствующая детерминированная спектральная задача. Запоминаются согласующиеся с выбранным вектором формы значения $\tilde{w}$ или - $\tilde{w}$ и отвечающие им собственные числа. После перебора всех возможных значений вектора формы, которые составляют $2^{m-1}$ вариантов, выбирается наименьшее собственное число и отвечающая ему собственная форма.

Евгений Илъич Михайловский (д.ф.-м.н., профессор), зав. кафедрой, каф. математического моделирования и кибернетики. Елена Владимировна Тулубенская, ст. преподаватель, каф. математического моделирования и кибернетики. 
Сформулированный алгоритм полного перебора вариантов (ППВ) всегда приводит к искомому решению. Однако он наталкивается на т. н. «проклятие размерности» (curse of dimensionality, Bellman, R. Е.): при использовании этого алгоритма, например, на сетке размерностью $m=100$ потребовалось бы решать свыше $10^{30}$ линейных спектральных задач.

Обойти названное препятствие в реализации алгоритма перебора вариантов позволяет предложенный в работе [1] комбинированный алгоритм «ППВ + ЛПВ» (ЛПВ - локальный перебор вариантов). Сначала на редкой сетке, т. е. такой, чтобы число $2^{m-1}$ было не слишком большим, реализуется алгоритм ППВ, и устанавливается отвечающая минимальному собственному числу $к a-$ чественно адекватная собственная форма, т. е. имеющая устойчивый с ростом $m$ вид графика. А затем при последовательном удвоении числа интервалов сетки выполняется перебор вариантов лишь вблизи корней названной формы, т. е. реализуется алгоритм ЛПВ.

В данной работе предлагается альтернативный комбинированному алгоритм движения по параметру жёсткости одной из разномодульных упругих сред. Поясним этот алгоритм на примере продольно сжимаемого шарнирно опёртого стержня на границе винклеровых сред с параметрами жёсткости $k_{1}=20, k_{2}=25$. Сначала рассматриваем случай однородной упругой среды с параметрами жёсткости $k_{1}=k_{2} \triangleq k=20$. Собственные числа для этого случая определяются по формуле [2]

$$
\lambda_{(n)}=n^{2}+\frac{k}{n^{2}},
$$

где $n$ - число полуволн собственной формы

$$
w(\xi)=B \sin n \xi, \xi \in[0, \pi] .
$$

Выясним, какому $n$ отвечает минимальное собственное число $\lambda_{1}$ при $k=$ $=20$. На основании формулы (3) получаем $\lambda_{(1)}=21, \lambda_{(2)}=9, \lambda_{(3)}=11,22$, $\lambda_{(4)}=17,25$. Отсюда следует, что $\lambda_{1}=\lambda_{(2)}=9, \lambda_{2}=\lambda_{(3)}=11,22, \lambda_{3}=\lambda_{(4)}=$ $=17,25$ и т. д. Таким образом, минимальному собственному числу $\left(\lambda_{1}=9\right)$ отвечает двухполуволновая собственная форма.

Далее решаем эту же задачу с использованием конечно-разностной аппроксимации на достаточно густой сетке, например, $m=$ $=100$. Получаем в рассматриваемом частном случае $\lambda_{1}=9,0065$. Соответствующую этому числу собственную форму рассматриваем как качественно адекватную для случая $k_{1}=20, k_{2}=21$. Используя формальный перебор вариантов, будем проверять на соответствие следующие векторы

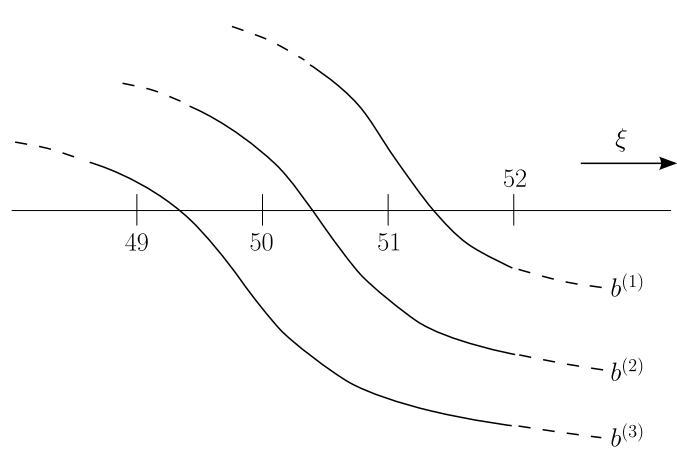

Рис. 1 формы (рис. 1):

$$
b^{(1)}=\left(b_{i}=1, i \in\{1,2, \ldots, 51\} ; b_{i}=0, i \in\{52,53, \ldots, 99\}\right) ;
$$




$$
\begin{aligned}
& b^{(2)}=\left(b_{i}=1, i \in\{1,2, \ldots, 50\} ; b_{i}=0, i \in\{51,52, \ldots, 99\}\right) ; \\
& b^{(3)}=\left(b_{i}=1, i \in\{1,2, \ldots, 49\} ; b_{i}=0, i \in\{50,51, \ldots, 99\}\right) .
\end{aligned}
$$

В случае $k_{1}=20, k_{2}=21$ век-

Таблица 1 торы формы $b^{(1)}, b^{(3)}$ не приво-

\begin{tabular}{c|c|c|c|c|c}
\hline$k_{2}$ & 21 & 22 & 23 & 24 & 25 \\
\hline$b^{(i)}$ & $b^{(2)}$ & $b^{(2)}$ & $b^{(2)}$ & $b^{(1)}$ & $b^{(1)}$ \\
\hline$\lambda_{1}$ & 9,129 & 9,243 & 9,346 & 9,443 & 9,532 \\
\hline
\end{tabular}
дят к согласованному решению, а варианту $b^{(2)}$ отвечает решение $\lambda_{1}=9,129$. Далее рассматриваем случай $k_{1}=20, k_{2}=22$, принимая для него за качественно адекватную форму, отвечающую случаю $k_{1}=$ $=20, k_{2}=21$. Вариант $b^{(2)}$ даёт решение $\lambda_{1}=9,243$. Продолжая процесс, получаем результаты, представленные в табл. 1.

1. Постановка спектральной задачи. Уравнение продольно-поперечного изгиба стержня имеет вид [3]

$$
E I w^{I V}=q-h_{\psi}^{2} q^{\prime \prime}-P w^{\prime \prime},
$$

где $E I$ - жёсткость стержня при изгибе; $q$ - погонная поперечная нагрузка; $P$ - продольная сжимающая сила; $\tilde{h}_{\psi}^{2}=E I / \mu S ; \mu=E / 2(1+\nu), E, \nu$ - модуль Юнга и коэффициент Пуассона материала стержня; $S$ - площадь поперечного сечения стержня; $w^{\prime}=d w / d x$.

Уравнение (5) является уравнением Эйлера для функционала

$$
J(w)=\int_{0}^{l}\left(\frac{1}{2} E I w^{\prime \prime 2}-\frac{1}{2} P w^{\prime 2}-q w+\tilde{h}_{\psi}^{2} q^{\prime \prime} w\right) d x .
$$

Впредь считаем, что ось стержня расположена на границе винклеровых сред с жёсткостями $c_{1}$ при $w>0$ и $c_{2}$ при $w<0$. Тогда в соответствии с аксиомой связей имеет место

$$
q=-c_{1} w_{+}-c_{2} w_{-},
$$

где $w_{+}, w_{-}$- положительная и отрицательная срезки функции прогиба:

$$
w_{+}=\left\{\begin{array}{ll}
w, & w>0, \\
0, & w \leqslant 0 ;
\end{array} \quad w_{-}= \begin{cases}0, & w \geqslant 0 \\
w, & w<0\end{cases}\right.
$$

Подставив вместо $q$ в уравнение (5) правую часть формулы (7), получим

$$
E I w^{I V}+c_{1} w_{+}+c_{2} w_{-}-h_{\psi}^{2}\left(c_{1} w_{+}+c_{2} w_{-}\right)^{\prime \prime}=-P w^{\prime \prime} .
$$

Ограничимся рассмотрением одинаковых граничных условий на краях $x=0$ и $x=l$, а именно граничных условий шарнирного опирания и жёсткой заделки.

В первом случае граничные условия имеют вид [3]

$$
w=0, \quad M=E I\left(-w^{\prime \prime}+\psi^{\prime}\right)=0 \text { при } x=0, x=l,
$$

где $\psi$ - поперечный сдвиг по модели С. П. Тимошенко. 
Принимая во внимание формулу $\psi^{\prime}=-q / \mu S$ и равенство (7), второе условие (9) можно преобразовать так:

$$
w^{\prime \prime}-\psi^{\prime}=w^{\prime \prime}-\frac{1}{\mu S}\left(c_{1} w_{+}+c_{2} w_{-}\right)=w^{\prime \prime}=0 .
$$

Таким образом, граничные условия шарнирного опирания при поперечной нагрузке в виде реакций винклеровой среды записываются так же, как и в случае классической теории изгиба балок:

$$
w=0, \quad w^{\prime \prime}=0 \text { при } x=0, x=l .
$$

Во втором случае граничные условия при учете поперечных сдвигов имеют вид

$$
w=0, \quad \vartheta \triangleq-w^{\prime}+\psi=0 \text { при } x=0, x=l .
$$

Исключить из второго граничного уравнения (11) сдвиг не представляется возможным. Поэтому ниже будем рассматривать граничные условия типа жёсткой заделки:

$$
w=0, \quad w^{\prime}=0 \text { при } x=0, x=l,
$$

т. е. такие же, как и в случае классической теории изгиба балок.

Выполним в уравнении (8) и в функционале (6) при учете соотношения (7) замену по формулам

$$
\xi=\frac{\pi}{l} x \in[0, \pi], \quad k_{i}=\frac{l^{4} c_{i}}{\pi^{2} E I}, \quad i=1,2 ; \quad \lambda=\frac{l^{2} P}{\pi^{2} E I}, \quad \varkappa^{2}=\frac{\pi^{2} h_{\psi}^{2}}{l^{2}} .
$$

Обозначая теперь штрихом производную по $\xi$ и записывая функционал с точностью до постоянного множителя, будем иметь

$$
\begin{gathered}
w^{I V}+k_{1} w_{+}+k_{2} w_{-}-\varkappa^{2}\left(k_{1} w_{+}+k_{2} w_{-}\right)^{\prime \prime}=-\lambda w^{\prime \prime} \\
\tilde{J}(w)=\frac{1}{2} \int_{0}^{\pi}\left[w^{\prime \prime 2}+k_{1} w_{+}{ }^{2}+k_{2} w_{-}{ }^{2}+\varkappa^{2}\left(k_{1} w_{+}+k_{2} w_{-}\right) w^{\prime \prime}-\lambda w^{\prime 2}\right] d \xi
\end{gathered}
$$

Рассмотрим, в частности, случай $k_{1}=k_{2}=k$. Уравнение (12) для однородной винклеровой среды принимает такой вид:

$$
w^{I V}+\left(\lambda-\varkappa^{2} k\right) w^{\prime \prime}+k w=0 .
$$

Учитывая, что спектральная задача при $\varkappa^{2}=0$ и при граничных условиях (10) имеет решение (3), (4), сразу можно записать

$$
\lambda_{(n)}=n^{2}+\frac{k}{n^{2}}+\varkappa^{2} k, \quad w=B \sin n \xi .
$$

Выявим значимость связанного с учётом поперечных сдвигов последнего слагаемого в формуле для $\lambda_{(n)}$. Зададимся вопросом: при каком $n$ отношение

$$
P_{(n)}=\varkappa^{2} k / \lambda_{(n)}
$$

принимает максимальное значение? 
В результате элементарных преобразований устанавливаем, что $\max P_{(n)}$ достигается при $n=[\sqrt[4]{k}]$, где квадратные скобки означают целую часть заключенного в них числа. При этом само отношение (15) принимает вид

$$
\max P_{(n)}=\frac{\varkappa^{2} n^{2}}{2+\varkappa^{2} n^{2}}
$$

Если положить, например, $\varkappa^{2}=0,004, k=10^{4}$, то получим

$$
\max P_{(n)}=0,1667(16,67 \%) .
$$

2. Конечно-разностная аппроксимация. Заменим формулу (13) приближённой с использованием дискретного представления функции $w(\xi), \xi \in$ $[0, \pi]$, на сетке $w_{i} \triangleq w\left(\xi_{i}\right), i \in\{-1,0, \ldots, m+1\}, \xi_{i+1}=\xi_{i}+h, h=\pi /(m+2)$, $\xi_{-1}=0, \xi_{m+1}=\pi$. Интегралы вычисляем по формуле трапеций, производные аппроксимируем следующими конечно-разностными отношениями:

$$
\begin{gathered}
w^{\prime}\left(\xi_{i}\right)=\frac{w_{i+1}-w_{i-1}}{2 h}, \quad w^{\prime \prime}\left(\xi_{i}\right)=\frac{w_{i+1}-2 w_{i}+w_{i-1}}{h^{2}} \\
w^{\prime}\left(\xi_{-1}\right)=\frac{-3 w_{-1}+4 w_{0}-w_{1}}{2 h}, \quad w^{\prime}\left(\xi_{m+1}\right)=\frac{w_{m-1}-4 w_{m}+3 w_{m+1}}{2 h}, \\
w^{\prime \prime}\left(\xi_{-1}\right)=\frac{w_{-1}-2 w_{0}+w_{1}}{h^{2}}, \quad w^{\prime \prime}\left(\xi_{m+1}\right)=\frac{w_{m-1}-2 w_{m}+w_{m+1}}{h^{2}} .
\end{gathered}
$$

Значения срезок функции $w(\xi)$ в узлах сетки представляем соотношениями (cм. (2))

$$
w_{+}\left(\xi_{i}\right)=b_{i} w_{i}, \quad w_{-}\left(\xi_{i}\right)=\left(1-b_{i}\right) w_{i} .
$$

Граничные условия шарнирного опирания $w(0)=w(\pi)=0, w^{\prime \prime}(0)=$ $=w^{\prime \prime}(\pi)=0$ в терминах дискретных значений функции $w(\xi)$ можно записать так:

$$
w_{-1}=w_{m+1}=0, \quad w_{0}=0,5 w_{1}, \quad w_{m}=0,5 w_{m-1} .
$$

Аналогично граничные условия типа жёсткого защемления $w(0)=w(\pi)=$ $=0, w^{\prime}(0)=w^{\prime}(\pi)=0$ имеют вид

$$
w_{-1}=w_{m+1}=0, \quad w_{0}=0,25 w_{1}, w_{m}=0,25 w_{m-1} .
$$

С учётом равенств (17) энергию деформации винклеровых сред (с точностью до постоянного множителя) можно представить формулой

$$
\frac{h}{2} \sum_{i=1}^{m-1}\left[c_{i}\left(k_{1} b_{i}^{2}+k_{2}\left(1-b_{i}\right)^{2}\right)\right] w_{i}^{2} \triangleq \frac{1}{2} \tilde{w}^{\top} C \tilde{w},
$$

где $\tilde{w}=\left[w_{1}, w_{2}, \ldots, w_{m-1}\right]^{\top}, C=h \operatorname{diag}\left[c_{1} \bar{b}_{1}, \ldots, c_{m-1} \bar{b}_{m-1}\right], \bar{b}_{i} \triangleq k_{1} b_{i}+$ $+k_{2}\left(1-b_{i}\right)$. (В выражении для матрицы $C$ на основании $(2)$ вместо $b_{i}^{2},\left(1-b_{i}\right)^{2}$ записано $b_{i}, 1-b_{i}$ соответственно.) 
Функционал (13) в соответствии с формулами (16), (20) можно заменить следующим приближённым выражением:

$$
\tilde{J}(\tilde{w})=\frac{1}{2} \tilde{w}^{\top} A \tilde{w}+\frac{1}{2} \tilde{w}^{\top} C_{1} \tilde{w}-\frac{1}{2} \lambda \tilde{w}^{\top} Q \tilde{w},
$$

где

$$
\begin{aligned}
& A=\frac{1}{h^{3}}\left[\begin{array}{ccccccl}
a_{11} & a_{12} & 1 & & & & 0 \\
a_{21} & 6 & -4 & 1 & & & \\
1 & -4 & 6 & -4 & 1 & & \\
\cdots & \cdots & \cdots & \ldots & \ldots & \ldots & \ldots \\
& & 1 & -4 & 6 & -4 & 1 \\
& 0 & & 1 & -4 & 6 & a_{m-2, m-1} \\
& & & & 1 & a_{m-1, m-2} & a_{m-1, m-1}
\end{array}\right] \\
& Q=\frac{1}{4 h}\left[\begin{array}{ccccccc}
q_{11} & q_{12} & -1 & & & & 0 \\
q_{21} & 2 & 0 & -1 & & & \mathbf{0} \\
-1 & 0 & 2 & 0 & -1 & \ldots & \ldots \\
\cdots & \cdots & \cdots & \cdots & \cdots & \ldots & -1 \\
& & -1 & 0 & 2 & 0 & q_{m-2, m-1} \\
& \mathbf{0} & & -1 & 0 & 2 & q_{m-1, m-1}
\end{array}\right] \\
& C_{1}=C-\frac{\varkappa^{2}}{h}\left[\begin{array}{ccccc}
c_{11} \bar{b}_{1} & \frac{\bar{b}_{1}+\bar{b}_{2}}{2} & & & \\
\frac{\bar{b}_{1}+\bar{b}_{2}}{2} & -2 \bar{b}_{2} & \frac{\bar{b}_{2}+\bar{b}_{3}}{2} & & \mathbf{0} \\
\cdots & \cdots & \cdots & \ldots & \ldots \\
0 & & \frac{\bar{b}_{m-3}+\bar{b}_{m-2}}{2} & -2 \bar{b}_{m-2} & \frac{\bar{b}_{m-2}+\bar{b}_{m-1}}{2} \\
0 & & & \frac{\bar{b}_{m-2}+\bar{b}_{m-1}}{2} & c_{m-1, m-1} \bar{b}_{m-1}
\end{array}\right] \text {. }
\end{aligned}
$$

В матрицах в зависимости от вида граничных условий следует полагать:

$$
\begin{aligned}
& a_{11}=a_{m-1, m-1}=3,25, \quad a_{12}=a_{21}=a_{m-1, m-2}=a_{m-2, m-1}=-3,5 ; \\
& q_{11}=q_{m-1, m-1}=2,75, \quad q_{12}=q_{21}=q_{m-1, m-2}=q_{m-2, m-1}=-0,5 ; \\
& c_{1}=c_{11}=c_{m-1}=c_{m-1, m-1}=1,5
\end{aligned}
$$

при шарнирном опирании краев;

$$
\begin{aligned}
& a_{11}=a_{m-1, m-1}=4,4375, \quad a_{12}=a_{21}=a_{m-1, m-2}=a_{m-2, m-1}=-3,75 ; \\
& q_{11}=q_{m-1, m-1}=2,0625, \quad q_{12}=q_{21}=q_{m-1, m-2}=q_{m-2, m-1}=-0,25 ; \\
& c_{1}=c_{11}=c_{m-1}=c_{m-1, m-1}=1,0625
\end{aligned}
$$

при жёстком защемлении краев.

Необходимое условие минимума функционала (21) имеет такой вид:

$$
\nabla_{\tilde{w}} \tilde{J}(\tilde{w})=A \tilde{w}+C_{1} \tilde{w}-\lambda Q \tilde{w}=0 .
$$

При отсутствии симметрии балки относительно сечения $x=l / 2$, осуществляя переход от винклеровых сред с жескостями $k_{1}, k_{1}+z \Delta k$ к случаю $k_{1}, k_{1}+(z+1) \Delta k$, приходится выполнять локальный перебор вариантов вектора формы вслепую, например, по первой или второй схеме ЛПВ [1]. Однако 
в случае одинаковых граничных условий на краях $x=0, x=l$ число вариантов сокращается в связи с предсказуемостью эволюции собственной формы при увеличении жёсткости $k_{2}$. Например, во введении рассматривались три «возможных» варианта вектора формы $b^{(1)}, b^{(2)}, b^{(3)}$. Однако очевидно, что при увеличении жёсткости $k_{2}$ точка, являющаяся корнем собственной формы, может перемещаться по оси $\xi$ лишь вправо (см. рис. 1) и поэтому форма $b^{(3)}$ не является возможной.

Несложный анализ эволюции собственной формы позволяет минимизировать число вариантов вектора формы для симметричной относительно сечения $x=l / 2$ конструкции.

Пусть собственная форма при $k_{1}=k_{2} \triangleq k$ имеет $p=n-1$ корней и пусть при жёсткостях $k_{1}, k_{1}+z \Delta k j$-тый корень собственной формы принадлежит интервалу $\left(\xi_{s_{j}}, \xi_{s_{j}+1}\right)$. Тогда при переходе от случая $k_{1}, k_{1}+z \Delta k$ к случаю $k_{1}, k_{1}+(z+1) \Delta k$ достаточно рассмотреть следующие варианты:

- $p$ - чётное число:

$$
\begin{gathered}
b_{i}^{(1)}= \begin{cases}1, & i \in\left\{1,2, \ldots, s_{1}\right\} ; \\
0, & i \in\left\{s_{1}+1, s_{1}+2, \ldots, s_{2}\right\} ; \\
1, & i \in\left\{s_{2}+1, s_{2}+2, \ldots, s_{3}\right\} ; \\
0, & i \in\left\{s_{p-1}+1, s_{p-1}+2, \ldots, s_{p}\right\} ; \\
1, & i \in\left\{s_{p}+1, s_{p}+2, \ldots, m-1\right\} ;\end{cases} \\
b_{i}^{(2)}= \begin{cases}1, & i \in\left\{1,2, \ldots, s_{1}+1\right\} ; \\
0, & i \in\left\{s_{1}+2, s_{1}+3, \ldots, s_{2}-1\right\} ; \\
1, & i \in\left\{s_{2}, s_{2}+1, \ldots, s_{3}+1\right\} ; \\
0, & i \in\left\{s_{p-1}+2, s_{p-1}+3, \ldots, s_{p}-1\right\} ; \\
1, & i \in\left\{s_{p}, s_{p}+1, \ldots, m-1\right\} ;\end{cases}
\end{gathered}
$$

- $p$ - нечётное число:

$$
\begin{gathered}
b_{i}^{(1)}= \begin{cases}1, & i \in\left\{1,2, \ldots, s_{1}\right\} ; \\
0, & i \in\left\{s_{1}+1, s_{1}+2, \ldots, s_{2}\right\} ; \\
1, & i \in\left\{s_{2}+1, s_{2}+2, \ldots, s_{3}\right\} ; \\
1, & i \in\left\{s_{p-1}+1, s_{p-1}+2, \ldots, s_{p}\right\} ; \\
0, & i \in\left\{s_{p}+1, s_{p}+2, \ldots, m-1\right\} ;\end{cases} \\
b_{i}^{(2)}= \begin{cases}1, & i \in\left\{1,2, \ldots, s_{1}+1\right\} ; \\
0, & i \in\left\{s_{1}+2, s_{1}+3, \ldots, s_{2}-1\right\} ; \\
1, & i \in\left\{s_{2}, s_{2}+1, \ldots, s_{3}+1\right\} ; \\
1, & i \in\left\{s_{p-1}+2, s_{p-1}+3, \ldots, s_{p}-1\right\} ; \\
0, & i \in\left\{s_{p}+2, s_{p}+3, \ldots, m-1\right\} .\end{cases}
\end{gathered}
$$


3. Примеры применения алгоритма движения по параметру жёсткости. Рассмотрим задачу об устойчивости шарнирно опёртого стержня на границе винклеровых сред. Нетрудно убедиться, что при $\varkappa^{2}=0, k=36$ минимальному собственному числу отвечают две собственные формы, так как $\lambda_{1}=\lambda_{(2)}=$ $=\lambda_{(3)}=13$ (см. (3)). Поэтому, задавшись целью исследовать эволюцию трёхполуволновой собственной формы при $k_{2} \rightarrow \infty$, примем $k_{1}=37$. Тогда в случае однородной винклеровой среды $k_{1}=k_{2}=37$ в соответствии с (14) при $\varkappa^{2}=0,004$ получим $\lambda_{(1)}=38,15, \lambda_{(2)}=13,40, \lambda_{(3)}=13,26, \lambda_{(4)}=18,46$. Таким образом, имеем $\lambda_{1}=\lambda_{(3)}=13,26$. В этом случае $p=2$ и формулы $(23)$ принимают такой вид (см. рис. 2):

$$
\begin{aligned}
& b_{i}^{(1)}= \begin{cases}1, & i \in\left\{1,2, \ldots, s_{1}\right\} ; \\
0, & i \in\left\{s_{1}+1, s_{1}+2, \ldots, s_{2}\right\} ; \\
1, & i \in\left\{s_{2}+1, s_{2}+2, \ldots, m-1\right\} ;\end{cases} \\
& b_{i}^{(2)}= \begin{cases}1, & i \in\left\{1,2, \ldots, s_{1}+1\right\} ; \\
0, & i \in\left\{s_{1}+2, s_{1}+3, \ldots, s_{2}-1\right\} ; \\
1, & i \in\left\{s_{2}, s_{2}+1, \ldots, m-1\right\} .\end{cases}
\end{aligned}
$$

Приближённое решение на основе конечно-разностной аппроксимации при $m=100$ для случая $k_{1}=k_{2}=37, \varkappa^{2}=0,004$ имеет вид $\lambda_{1}=\lambda_{(3)}=13,286$. При этом корни собственной формы содержатся в интервалах $\left(\xi_{33}, \xi_{34}\right)$, $\left(\xi_{66}, \xi_{67}\right)$ и формулы принимают такой вид:

$$
b_{i}^{(1)}=\left\{\begin{array}{ll}
1, & i \in\{1,2, \ldots, 33\} ; \\
0, & i \in\{34,35, \ldots, 66\} ; \\
1, & i \in\{67,68, \ldots, 99\} ;
\end{array} \quad b_{i}^{(2)}= \begin{cases}1, & i \in\{1,2, \ldots, 34\} ; \\
0, & i \in\{35,36, \ldots, 65\} ; \\
1, & i \in\{66,67, \ldots, 99\} .\end{cases}\right.
$$

Выборочные результаты расчётов представлены в виде табл. 2 и рис. 3.

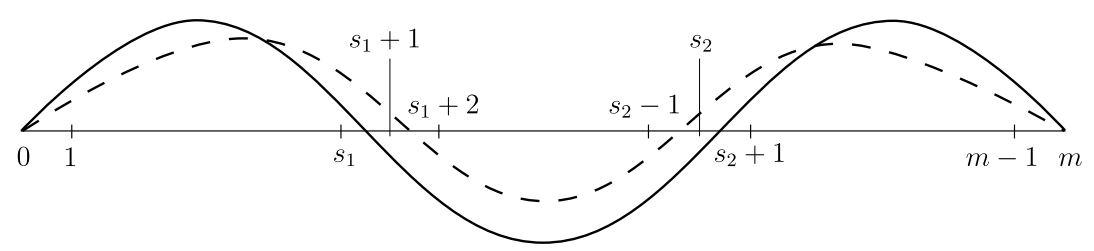

\begin{tabular}{|c|c|c|c|c|c|}
\hline$k_{2}$ & 37 & 50 & 60 & 80 & 100 \\
\hline$\lambda$ & 13,286 & 13,681 & 13,888 & 14,174 & 14,366 \\
\hline Собственная форма & 1 & 2 & 3 & 4 & 5 \\
\hline 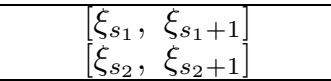 & $\begin{array}{ll}\xi_{33}, & \xi_{34} \\
{\left[\xi_{66},\right.} & \xi_{67}\end{array}$ & $\begin{array}{ll}\xi_{34}, & \xi_{35} \\
{\left[\xi_{65},\right.} & \xi_{66}\end{array}$ & $\begin{array}{ll}\xi_{35}, & \xi_{36} \\
{\left[\xi_{64},\right.} & \xi_{65}\end{array}$ & $\begin{array}{ll}\xi_{36}, & \xi_{37} \\
{\left[\xi_{63},\right.} & \xi_{64}\end{array}$ & $\begin{array}{ll}\xi_{37}, & \xi_{38} \\
{\left[\xi_{62},\right.} & \xi_{63}\end{array}$ \\
\hline
\end{tabular}

Рис. 2

Таблица 2 


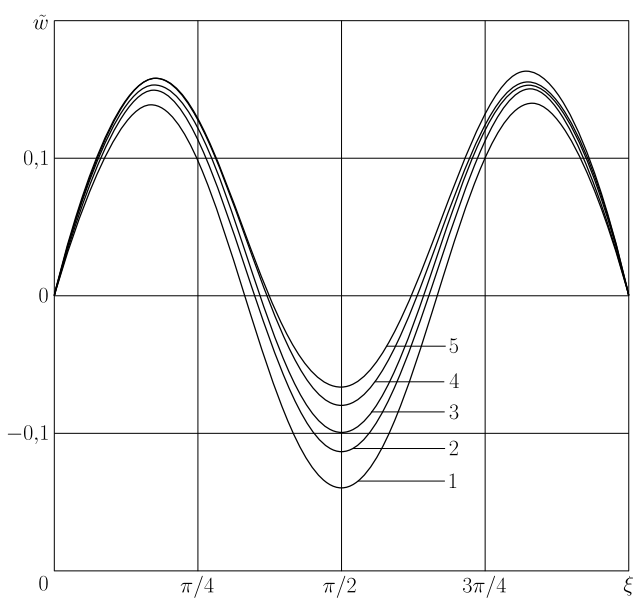

Рис. 3

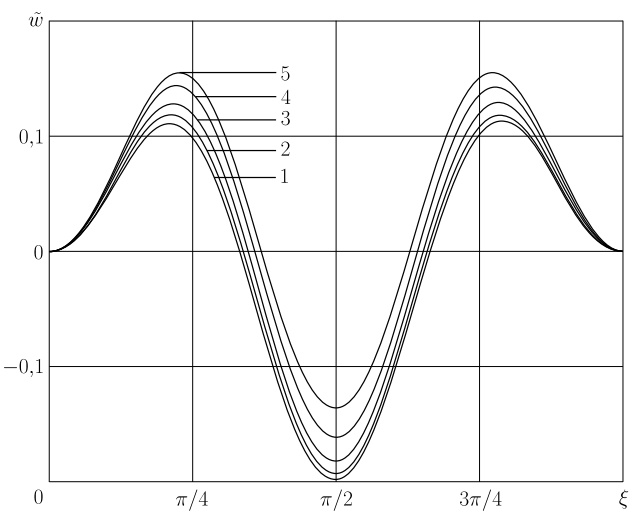

Рис. 4

Рассмотрим теперь анаТаблица 3 логичную задачу для жёст-

\begin{tabular}{c|c|c|c}
\hline$k$ & $\lambda$ & $p$ & Вектор формы \\
\hline 62 & 19,975 & 1 & $b_{i}= \begin{cases}1, & i \in\{1,2, \ldots, 50\} \\
0, & i \in\{51,52, \ldots, 99\}\end{cases}$ \\
63 & 20,137 & 1 & $b_{i}= \begin{cases}1, & i \in\{1,2, \ldots, 50\} \\
0, & i \in\{51,52, \ldots, 99\}\end{cases}$ \\
64 & 20,299 & 1 & $b_{i}= \begin{cases}1, & i \in\{1,2, \ldots, 50\} \\
0, & i \in\{51,52, \ldots, 99\}\end{cases}$ \\
65 & 20,414 & 2 & $b_{i}= \begin{cases}1, & i \in\{1,2, \ldots, 33\} \\
0, & i \in\{34,35, \ldots, 66\} \\
1, & i \in\{67,68, \ldots, 99\}\end{cases}$ \\
66 & 20,518 & 2 & $b_{i}= \begin{cases}1, & i \in\{1,2, \ldots, 33\} \\
0, & i \in\{34,35, \ldots, 66\} \\
1, & i \in\{67,68, \ldots, 99\}\end{cases}$ \\
\hline
\end{tabular}
ко защемленного стержня. Как известно [2], в случае жёстко защемленного стержня использование аналитического решения сопряжено с выполнением громоздких численных расчетов. Поэтому для установления значений параметра жесткости $k=k_{1}=k_{2}$, при котором на смену собственной формы, имеющей одну точку пересечения с осью $\xi$, приходит форма с двумя точками пересечения (условнотрехполуволновая), проводился численный эксперимент. Некоторые результаты этого эксперимента приведены в табл. 3.

Далее применялся алгоритм движения по параметру жёсткости, начиная с $k=65$. Результаты расчетов приведены в табл. 4 и показаны на рис. 4.

Таблица 4

\begin{tabular}{|c|c|c|c|c|c|}
\hline$k_{2}$ & 65 & 70 & 80 & 100 & 130 \\
\hline$\lambda$ & 20,414 & 20,731 & 21,276 & 22,124 & 23,016 \\
\hline Собственная форма & 1 & 2 & 3 & 4 & 5 \\
\hline $\begin{array}{l}{\left[\xi_{s_{1}}, \xi_{s_{1}+1}\right]} \\
{\left[\xi_{s_{2}}, \xi_{s_{2}+1}\right]}\end{array}$ & \begin{tabular}{|ll}
$\xi_{33}$, & $\xi_{34}$ \\
{$\left[\xi_{66}\right.$,} & $\xi_{67}$
\end{tabular} & \begin{tabular}{|ll}
$\xi_{33}$, & $\xi_{34}$ \\
{$\left[\xi_{66}\right.$,} & $\xi_{67}$ \\
\end{tabular} & $\begin{array}{ll}\xi_{34}, & \xi_{35} \\
{\left[\xi_{65},\right.} & \xi_{66} \\
\end{array}$ & $\begin{array}{ll}\xi_{35}, & \xi_{36} \\
{\left[\xi_{64},\right.} & \xi_{65} \\
\end{array}$ & $\begin{array}{ll}\xi_{36}, & \xi_{37} \\
{\left[\xi_{63},\right.} & \xi_{64} \\
\end{array}$ \\
\hline
\end{tabular}

Работа выполнена при поддержке РФФИ (проект № 09-01-00178-а) и Федеральной целевой программы «Научные и научно-педагогические кадры инновационной России» на 2009-2013 годы (ГЗ № 02.740.11.0618). 


\section{БИБЛИОГРАФИЧЕСКИЙ СПИСОК}

1. Михайловский Е. И., Тулубенская Е. В. Алгоритм локального перебора вариантов в одной существенно нелинейной спектральной задаче // ПММ, 2010. Т. 14, № 2. С. 299-310; англ. пер.: Mikhailovskii E.I., Tulubenskaya E.V. An algorithm for the local exhaustive search for alternatives in an essentially non-linear eigenvalue problem // J. Appl. Math. Mech., 2010. Vol. 74, no. 2. Pp. 214-222.

2. Вольмир А.С. Устойчивость деформируемых систем. М.: Наука, 1967. 984 с. [Vol'mir A. S. Stability of Deformable Systems. Moscow: Nauka, 1967. 984 pp.]

3. Михайловский Е.И., Тарасов В.Н. Конструктивно-нелинейная механика пластин и оболочек// Вестн. Сыкт. ун-та. Сер. 1. Мат. Мех. Инф., 2010. №12. С. 3-32. [Mikhailovskii E.I., Tarasov V.N. Constructive-nonlinear mechanics of plates and shells // Vestn. Sykt. un-ta. Ser. 1. Mat. Meh. Inf., 2010. no.12. Pp. 3-32].

MSC: 74H15; 74G60, 74B20

\section{ALGORITHM OF MOTION ON THE PARAMETER OF RIGIDITY IN STABILITY PROBLEM ON THE BORDER OF WINKLER'S MEDIUMS}

\section{E. I. Mikhailovskii, E. V. Tulubenskaya}

Syktyvkar State University, Faculty of Mathematics, 55, Oktyabrskii pr., Syktyvkar, 167001, Russia.

E-mails: emich@inbox.ru, tulubenskaya@bk.ru

To solve the stability problem of one-dimensional elements of construction on the border of different elastic ambiences the algorithm of motion on the parameter of one ambience is suggested. Evolution of triple half-wave eigenform of longitudinal compressed rod is studied according to S. P. Timoshenko model's using boundary conditions of articulate and rigid fixing.

Key words: stability, spectral problem, brute-force search.

Original article submitted $07 / \mathrm{XI} / 2010$; revision submitted 17/VIII/2011.

Evgeniy I. Mikhailovskii (Dr. Sci. (Phys. \& Math.)), Head of Dept., Dept. of Mathematical Model \& Cybernetics. Elena V. Tulubenskaya, Senior Teacher, Dept. of Mathematical Model \& Cybernetics. 